\title{
Preface to Special Issue on Environmental and Energy Catalysis for Sustainable Development
}

Environmental pollution and energy shortage has been regarded as the two major challenges because of the rapid development of urbanization and industrialization. Considering these challenges, providing green environment and energy for human beings are pivotal for future sustainable development. Nanostructured catalysts (photocatalysts, thermal catalysts and electrocatalysts) with unique physiochemical properties could offer numerous opportunities to solve these issues of environmental and energetic sustainability. In recent years, significant advances have been made on the synthesis, mechanistic understanding and innovative applications of the new catalysts for environmental and energetic problems. These new catalysts have found wide applications in different fashions. The structural features of the catalytic materials can be further tuned via specific methods to allow for enhanced catalytic performance in tackling the challenges. The rapid development in catalysis science and technology has significantly promoted our understanding on the controlled synthesis, catalysis mechanism, and structure-activity relationship of various catalytic materials.

The recent rapid development in the area of environmental and energy catalysis for sustainability has inspired us to launch this special issue. We have invited emerging scientists to contribute original research articles that could improve our understanding of the key scientific and technological problems of nanostructured catalysts as applied for sustainability. The original articles describing catalytic applications for environmental remediation (air pollution control, wastewater treatment, disinfection), and for green energy have been accepted for publication. In this special issue, the readers will find very interesting contents covering the following aspects: (1) design and synthesis of catalytic materials with exposed active sites; (2) photocatalysts for wastewater treatment and air pollution control; (3) thermal catalysts for air pollutants decomposition; (4) mechanistic and theoretical understanding of the catalysis process as applied in environmental problems; (5) new concepts of photo-Fenton and plasmon catalysis.

At last, as the Guest Editors of this special issue, we would like to express our sincere appreciation to all the invited authors who contributed to this issue and all the reviewers for their valuable comments on the submitted manuscripts. We hope that the readers will find the articles in this special issue interesting and useful. Especially, we appreciate the editorial office staff of Chinese Journal of Catalysis for their hard work in editing and publishing this topic issue.

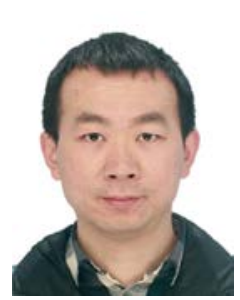

Prof. Fan Dong

Research Center for Environmental Science \& Technology, Institute of Fundamental and Frontier Sciences, University of Electronic Science and Technology of China E-mail: dfctbu@126.com

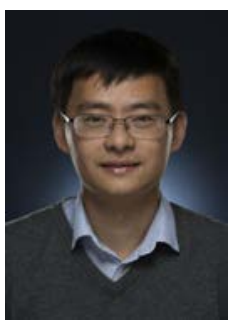

Prof. Ying Zhou

State Key Laboratory of Oil and Gas Reservoir Geology and Exploitation, School of Materials Science and Engineering, Southwest Petroleum University, China E-mail:yzhou@swpu.edu.cn

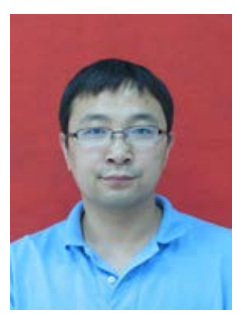

Prof. Guidong Yang XJTU-Oxford Joint International Research Laboratory of Catalysis, School of Chemical Engineering and Technology, Xi'an Jiaotong University, Xi'an 710049, China E-mail: guidongyang@xjtu.edu.cn 